\title{
Sobre ensambles y ensamblajes ecológicos - respuesta a Monge-Nájera
}

\author{
Alonso Ramírez ${ }^{1} \&$ Pablo E. Gutiérrez-Fonseca ${ }^{2}$ \\ 1. Departamento de Ciencias Ambientales, Universidad de Puerto Rico, San Juan, Puerto Rico 00931; \\ aramirez@ramirezlab.net \\ 2. Departamento de Biología, Universidad de Puerto Rico, San Juan, Puerto Rico 00931; \\ gutifp@gmail.com
}

Recibido 29-IX-2015. Corregido 10-XI-2015. Aceptado 11-XII-2015.

\begin{abstract}
Ecological ensembles and assemblages - a reply to Monge-Nájera. Critical evaluation of terminology use in ecology is important to avoid using jargon that helps little to facilitate communication. Here we reply to Monge-Najera's comment on the use of the terms ensemble and assemblage, we argue for the use of the words ensamble and ensamblaje in Spanish. In addition, both terms have a proper place in ecology and we provide a case for their use in proper ecological context. Rev. Biol. Trop. 64 (2): 817-819. Epub 2016 June 01.
\end{abstract}

Key words: assemblage, ensemble, terms.

El campo de las ciencias está lleno de terminologías, algunas más o menos aceptadas o acertadas. El ejercicio de cuestionarnos la terminología que usamos es importante y nos permite evitar caer en lenguajes complejos que aportan poco a una comunicación clara. Monge-Nájera (2015) aborda dos términos que han cobrado popularidad en años recientes, ensamble y ensamblaje. En su trabajo, Monge-Nájera argumenta que dichos términos no son recomendados, por ser adaptaciones de términos en otros lenguajes, por lo que sugiere conjunto y asamblea como reemplazo de ensamble y ensamblaje, respectivamente. Además, alega que a pesar de que dichas palabras tienen su espacio dentro de la ecología, en muchos casos se puede prescindir de su uso. En nuestra opinión, estos términos tienen un uso importante dentro de la ecología y coincidimos con Monge-Nájera en que en algunos casos no son usados de forma apropiada. El propósito de esta nota es abogar por el uso de ensamble y ensamblaje en un contexto apropiado, de forma que faciliten la comunicación en lugar de dificultar o, en algunos casos, confundir la lectura.
En lo que respecta a los términos usados, diferimos de la propuesta de Monge-Nájera de usar conjunto y asamblea. Las palabras ensamble y ensamblaje son términos válidos y son frecuentemente usados en otros contextos (e.g., un ensamble de cuerdas). Consultamos la Real Academia de la Lengua Española (RAE) con el fin de salir de cualquier duda. El departamento de "Español al día" nos informó que "no hay motivos para censurar el empleo de términos como ensamble, ensamblaje o ensambladura, derivados correctos formados a partir del verbo ensamblar. Son voces empleadas en otros ámbitos además del de la ecología, siempre con un sentido general similar al de grupo o conjunto." Conjunto y asamblea transmiten la misma idea general. Sin embargo, ensamble y ensamblaje reflejan un aspecto adicional que consideramos importante: las piezas interactúan entre ellas. Ambas palabras transmiten un significado más preciso de lo que queremos describir. Un ensamblaje de insectos acuáticos, por ejemplo, no es un conjunto de insectos nada más. Es un grupo de organismos que interactúan entre ellos de una forma específica y participan de procesos ecosistémicos. 
En ecología raras veces trabajamos con una comunidad completa. Al menos en el sentido estricto del término comunidad. Los estudios generalmente se enfocan en componentes de la comunidad. Por ejemplo, en nuestro laboratorio estudiamos los ecosistemas de ríos, nuestras investigaciones generalmente se enfocan en los invertebrados, por lo que excluimos otros miembros de la comunidad (e.g., los peces). Aquí coincidimos con Monge-Nájera; si trabajamos con invertebrados, no debemos decir "la comunidad de invertebrados", hay que ser claros en que el trabajo solo mira una parte de la comunidad. Sin embargo, en aras de ser claros sobre el objetivo o tipo de estudio, creemos que es importante usar un término que llene el lugar del de "comunidad". Ensamble y ensamblaje llenan ese espacio.

Ensamblaje se refiere al estudio de una parte de la comunidad seleccionada desde un punto de vista taxonómico (Fauth et al. 1996). Cuando estudiamos solo las aves o los insectos dentro de una comunidad, estamos estudiando un ensamblaje. Ahora bien, al igual que el estudio de comunidades, el estudio de ensamblajes va más allá de una lista de especies. El objetivo del estudio debe contestar preguntas sobre cómo diferentes componentes interactúan entre sí y con su ambiente. El término ensamblaje busca transmitir información sobre cómo están estructurados los miembros del conjunto. Un estudio sobre la composición taxonómica y funcional, y la estructura trófica de los invertebrados de ríos urbanos, debería llevar un título como "características de los ensamblajes de invertebrados de ríos urbanos". Si solamente decimos "los invertebrados de ríos urbanos," se nos quedaría por fuera parte de la información, en particular la parte de función y estructura. Usando el término ensamblaje transmitimos la idea de que el estudio es mucho más que una lista de especies; se trata de un estudio en que usamos un enfoque de ecología de comunidades para estudiar un componente de la misma.

El término ensamble hace referencia a un grupo filogenéticamente relacionado que explota un recurso similar dentro de una comunidad (Fauth et al. 1996). A partir de esta definición se puede esperar que la interacción entre organismos resulte en determinadas interacciones ecológicas propias de las comunidades, como competencia, exclusión, facilitación, entre otras. Para caracterizar un ensamble es necesario clarificar los componentes que se van a estudiar: el grupo taxonómico, el recurso explotado y el área geográfica, de tal manera que cuando decimos "El ensamble de peces insectívoros de Puerto Rico" se describe un conjunto de especies que co-habita e interactúa dentro de un ensamblaje (i.e., peces), los cuales pertenece a un gremio (i.e., insectívoros) en un determinado lugar (i.e., Puerto Rico). Si únicamente hacemos referencia a "Los peces insectívoros de Puerto Rico" suprimimos información valiosa de cómo el ensamblaje se relaciona con los demás miembros del conjunto, el recurso y el ambiente.

Apoyamos el punto sobre evitar términos que compliquen innecesariamente los textos. Así que antes de escoger utilizar ensamblaje o ensamble, debemos preguntarnos si las mismas de verdad ayudan a aclarar el tema en cuestión. Los mismos se deben usar cuando queremos enfatizar que estamos investigando una parte de la comunidad y cómo los componentes de la misma interactúan entre ellos. Como argumenta Monge-Nájera, se puede prescindir de estos términos en estudios sobre listas de especies que no se enfoquen en caracterizar las interacciones entre los componentes y el ambiente.

El cuestionamiento de Monge-Nájera (2015) sobre el uso de ensamble y ensamblaje nos permite examinar el uso de estos términos en nuestros trabajos. Es un proceso valioso que nos ayuda a mantener claridad a la hora de diseñar y comunicar nuestros estudios ecológicos. Debemos siempre ser cuidadosos al usar terminología y aplicarla apropiadamente. Sobre ensamble y ensamblaje, concluimos que ambos términos tienen su espacio en la ecología y su uso es válido bajo las condiciones correctas. Esperamos que esta nota ayude a pensar con cuidado las terminologías que usamos en ecología. 


\section{AGRADECIMIENTOS}

A Rodolfo Novelo-Gutiérrez por comentarios en el manuscrito y a los revisores anónimos por sus evaluaciones críticas.

\section{RESUMEN}

Evaluar críticamente la terminología que usamos en ecología es importante para evitar el uso de palabras que no ayudan a facilitar la comunicación científica. En este trabajo contestamos el comentario de Monge-Najera sobre el uso de los términos ensamble y ensamblaje, argumentando que estas palabras son válidas y útiles en el idioma español.
Además, ambos términos tienen su lugar en la ecología, por lo que proveemos información para usarlos en un contexto ecológico apropiado.

Palabras clave: ensamblaje, ensamble, términos.

\section{REFERENCIAS}

Fauth, J. E., Bernardo, J., Camara, M., Resetarits, W. J., Van Buskirk, J., \& McCollum, S. A. (1996). Simplifying the jargon of community ecology: A conceptual approach. American Naturalist 147(2), 282-286.

Monge-Nájera, J. (2015). ¿Existen realmente los ensambles y ensamblajes ecológicos? Revista de Biología Tropical 63(3), 575-577. 
